\title{
Effects of medicinal plant extracts on gluconeogenesis
}

\author{
This article was published in the following Dove Press journal: \\ Botanics:Targets and Therapy \\ 16 June 2012 \\ Number of times this article has been viewed
}

\section{Adolfo Andrade-Cetto \\ Laboratorio de Etnofarmacología, Departamento de Biología Celular, Facultad de Ciencias, Universidad Nacional Autónoma de México, Coyoacán, México}

\begin{abstract}
On a global level, type 2 diabetes mellitus (T2DM) is the most common endocrine disorder. T2DM is defined as an elevated blood glucose level associated with the absence of or inadequacy in pancreatic insulin secretion. The liver plays a key role in maintaining blood glucose levels during fasting by synthesizing glucose, mainly from lactate and amino acids through a process called gluconeogenesis. Because hepatic glucose production is increased at least twofold in patients with T2DM, targeting this pathway may lead to a blood glucose reduction in these patients. Botanical agents show promise for the development of new compounds to treat T2DM. Important mechanisms of action function via the inhibition of gluconeogenesis can occur in one of five ways: direct enzyme inhibition; through the downregulation of mRNA levels of fructose-1,6-bisphosphatase and glucose-6-phosphatase (G-6-P); through the activation of AMP-activated protein kinase, which leads to decreased levels of cAMP response elementbinding protein, a key transcription factor for gluconeogenic enzyme phosphorylation; through the expression of the glucokinase gene, which stimulates glucokinase activity and inhibits G-6-P; and through the inhibition of phosphoenolpyruvate carboxykinase, which decreases gluconeogenesis and enzymatically inhibits G-6-P and fructose-1,6-diphosphatase.
\end{abstract}

Keywords: type 2 diabetes mellitus, medicinal plants, gluconeogenesis, glucose 6-phosphatase, hepatic glucose production, endocrine disorder, fructose 1,6-diphosphatase

\section{Introduction}

On a global level, type 2 diabetes mellitus (T2DM) is the most common endocrine disorder, and the World Health Organization estimates that by 2030, the number of diabetic people worldwide will increase $214 \%$ from the number of cases reported in $2000 .^{1}$

T2DM is defined as an elevated blood glucose level associated with the absence of or inadequate pancreatic insulin secretion, which may be expressed with or without concurrent impairment of insulin action. T2DM is also characterized by tissue resistance to insulin combined with this deficiency in insulin secretion. Although patients with insulin resistance continue to produce insulin in their beta cells, inadequate secretion causes blood glucose levels to increase. ${ }^{2}$

In T2DM, insulin resistance in the liver reflects the failure of hyperinsulinemia to suppress gluconeogenesis, resulting in fasting hyperglycemia and decreased liver glycogen storage in the postprandial state. Increased hepatic glucose production occurs early in the course of diabetes, although the increase is more pronounced after the onset of insulin secretory abnormalities and insulin resistance in skeletal muscle. ${ }^{3}$

Gluconeogenesis is important in preventing an excessive reduction in the blood glucose concentration during fasting. Glucose is the primary substrate for energy
Correspondence: Adolfo Andrade-Cetto Laboratorio de Etnofarmacología, Departamento de Biología Celular, Facultad de Ciencias, Universidad Nacional Autónoma de México, Coyoacán 045I0, D.F. México Email aac@ciencias.unam.mx 
in tissues, such as in the brain, and for the red blood cells; therefore, adequate amounts of glucose must be present in the blood for several hours between meals. The liver plays a key role in maintaining blood glucose levels during fasting by converting its stored glycogen into glucose (glycogenolysis) and by synthesizing glucose, mainly from lactate and amino acids (gluconeogenesis). ${ }^{4}$

It was demonstrated that hepatic glucose production is increased at least twofold in patients with T2DM. ${ }^{5}$ In T2DM, subjects with mild to moderate fasting hyperglycemia (ie, 140-200 mg/dL), basal hepatic glucose production is increased by approximately $0.5 \mathrm{mg} / \mathrm{kg}$ per minute. Consequently, during overnight sleeping hours (ie, 2200 to 0800 hours), the liver of an $80 \mathrm{~kg}$ diabetic individual with modest fasting hyperglycemia adds an additional $35 \mathrm{~g}$ of glucose to the systemic circulation. The increase in basal hepatic glucose production (HGP) is correlated closely with the severity of fasting hyperglycemia. Thus, in type 2 diabetics with overt fasting hyperglycemia (ie, $>140 \mathrm{mg} / \mathrm{dL}$ ), an excessive rate of hepatic glucose output is the major abnormality that causes elevated fasting plasma glucose levels. ${ }^{3}$

Recently, new targets for the management of T2DM have been proposed, and among them, the inhibitors of glucose6-phosphatase (G-6-P) have shown promise. ${ }^{6,7}$

\section{Hepatic targets for controlling T2DM}

The liver contributes to glucose homeostasis through rapid postprandial clearance of glucose from the portal vein in the absorptive state after a meal. When blood glucose falls below normal concentrations, glycogen is mobilized, and glucose is produced by gluconeogenesis. When blood glucose concentration increases, hepatic glucose uptake increases proportionally, stimulating glucokinase and glycogen synthesis. Elevated blood glucose concentrations normally increase insulin release and reduce glucagon release, increasing the insulin-to-glucagon ratio, which inactivates glycogen phosphorylase (inhibiting glycogenolysis), activates glycogen synthase (stimulating glycogen synthesis), and increases the concentrations of fructose-1,6-bisphosphate (FBP). These events reduce hepatic production of glucose and increase hepatic storage of glucose as glycogen.

The main enzymatic targets for controlling the elevation of blood sugar levels are the inhibition of G-6-P, the inhibition of FBP, and the inhibition of glycogen phosphorylase. ${ }^{6}$

\section{Inhibition of glucose-6-phosphatase}

G-6-P converts glucose-6-phosphate to glucose as a final step in glycogenolysis and gluconeogenesis. Thus, inhibition of this enzyme reduces hepatic glucose output and lowers glucose concentrations. Generally, the treatments used for diabetes management, such as metformin and insulin, can reduce the expression of G-6-P. ${ }^{6}$

In animal studies, inhibition of G-6-P rapidly reduces blood glucose concentrations. However, this strategy has two main limitations. First, given that G-6-P catalyzes the final step of glycogenolysis and gluconeogenesis, inhibition of this enzyme might precipitate hypoglycemia because it hinders the main counter-regulatory response triggered by glucagon and catecholamines. Second, the accumulation of glucose-6-phosphate, resulting from G-6-P inhibition, has been implicated in the induction of lipogenic genes that leads to hepatic steatosis. ${ }^{7}$

Unlike the inhibition of G-6-P, inhibition of FBP does not induce hypoglycemia because of a concomitant increase in glycogenolysis that does not increase glucose-6-phosphate concentrations. Thus, inhibition of FBP does not cause hepatic steatosis.?

\section{AMP-activated protein kinase}

In addition to direct enzyme inhibition, another pathway to suppress gluconeogenesis in the liver is the activation of AMP-activated protein kinase (AMPK), which directly phosphorylates its substrates, including glycogen synthase kinase $3 \beta$, cAMP response element-binding (CREB) protein- and CREB-regulated transcription coactivator 2 (CRTC2). AMPK achieves its downstream effects by direct phosphorylation of immediate substrates as well as through long-term effects on gene expression. As a molecular mechanism underlying AMPK-mediated phosphoenolpyruvate carboxykinase gene suppression, the phosphorylation of CRTC 2 by AMPK has been reported. ${ }^{8}$

Other studies suggest that AMPK induces orphan nuclear receptor small heterodimer partner gene expression and inhibits hepatic gluconeogenic gene expression in animal models. ${ }^{9}$ Small heterodimer partner decreases CREB-dependent induction of gluconeogenic gene expression and hepatic glucose production via disruption of the CREB-CRTC2 complex due to direct interaction with CREB. ${ }^{10}$

\section{Hepatic action of metformin}

Metformin is widely used for the therapy of T2DM. It lowers blood glucose levels without causing overt hypoglycemia or stimulating insulin secretion. In a study of the metformin mechanism of action, it was postulated that metformin decreases endogenous glucose production in T2DM patients. The increased plasma glucose level was attributed to a 
threefold increase in the rate of gluconeogenesis, as assessed by nuclear magnetic resonance spectroscopy. ${ }^{4}$ Metformin treatment decreased fasting plasma glucose concentrations by $25 \%$ and reduced glucose production. The drug decreased hepatic gluconeogenesis by interfering with respiratory oxidation in mitochondria. It suppressed gluconeogenesis from several substrates, including lactate, pyruvate, glycerol, and amino acids. In addition, metformin increases intramitochondrial levels of calcium ( $\mathrm{Ca}++)$, a modulator of mitochondrial respiration. ${ }^{11}$

Furthermore, other hypotheses have been proposed to explain the beneficial effects of metformin. In a study that examined the molecular mechanism through which metformin exerts its effect, the investigators found that metformin activates AMPK in primary rat hepatocytes, but has no direct effect on the partially purified enzyme in an in vitro kinase assay. ${ }^{12}$ This finding suggests that metformin indirectly activates AMPK by acting on a target other than AMPK itself. After AMPK activation, a variety of downstream biochemical changes occur, including decreased hepatic fatty acid synthesis. In addition, very low density lipoprotein synthesis decreases as a result of reduced acetylCoA carboxylase activity. AMPK activation thus results in the suppression of hepatic glucose production and increased glucose uptake in skeletal muscle.

\section{Effect of botanicals on gluconeogenesis}

As mentioned previously, metformin acts on the liver to produce a hypoglycemic effect. Metformin is a biguanide derivate from the active ingredient of the French lilac galegine or isoamylene guanidine. The plant Galega officinalis was prescribed in medieval times to relieve the intense urination accompanying diabetes mellitus. ${ }^{13}$ In recent years, some researchers have focused their attention on the role that botanicals play in the inhibition of gluconeogenesis. In a previous work from 2008 that reviews the mechanism of action of medicinal plants used in the treatment of T2DM, ${ }^{14}$ the following species are mentioned as inhibitors of the FBP enzyme: Aegle marmelos (L) Correa ex Roxb; Casearia esculenta (Roxb); Eclipta alba (L); Murraya koenigii (L); Ocimum sanctum (L); Piper betle (L); Trigonella foenum-graecum (L); and Coccinia indica Wight and Arn. Meanwhile, the following species inhibit G-6-P: Aegle marmelos (L) Correa ex Roxb; Allium sativum (L); Allium cepa (L); Casearia esculenta Roxb; Coscinium fenestratum Colebr; Eclipta alba (L); Eugenia jambolana (L); Eucommia ulmoides Oliv; Momordica charantia (L); Murraya koenigii (L); Ocimum sanctum (L); Piper betle (L); Pterocarpus marsupium Roxb; Tinospora cordifolia (Willd.) Hook f and Thomson; Aconitum carmichaelii Debeaux; Coccinia indica Wight and Arn; Enicostemma littorale Blume; and Syzygium aromaticum (L) Merrill and Perry.

Because gluconeogenesis inhibition is a promising strategy for new therapeutic agents, the present manuscript reviews how researchers working with hypoglycemic plants are approaching this topic, with the aim of encouraging future researchers to identify active botanicals or natural products that could act on this target. The manuscript covers work carried out since 2009. Examples of each approach are mentioned in Table 1.

\section{Artemisia sacrorum Ledeb (Asteraceae)}

This plant is used in traditional Asian medicine to treat diverse chronic and acute hepatitis. Glucose production through AMPK activation in human HepG2 cells was investigated, and the authors found that the petroleum ether fraction of the aerial part of the plant substantially inhibited glucose production in a concentration-dependent manner. The fraction induced the phosphorylation of AMPK, the downstream effector of acetyl-CoA carboxylase, and decreases the concentration of cAMP response elementbinding protein, a key transcription factor for gluconeogenic enzyme phosphorylation.

The extract also downregulated gluconeogenic gene expression of peroxisome proliferation activated receptor-y,

Table I Examples of hypoglycemic plants that can target hepatic glucose output

\begin{tabular}{ll}
\hline Plant & Activity \\
\hline Artemisia sacrorum & Inhibition of glucose production through the activation of AMPK \\
Cecropia obtusifolia & Inhibition of glucose-6-phosphatase \\
Malmea depressa & Inhibition of glucose-6-phosphatase \\
Melissa officinalis & Stimulation of glucokinase activity and inhibition of glucose-6-phosphatase \\
Salacia reticulate & Downregulation of genes involved in the gluconeogenic pathway \\
Satureja khuzestanica & Decreased gluconeogenesis by inhibition of phosphoenolpyruvate carboxykinase \\
Tinospora cordifolia & Inhibition of glucose 6-phosphatase and fructose I,6-diphosphatase
\end{tabular}

Abbreviation: AMPK, AMP-activated protein kinase. 
coactivator-1a phosphoenolpyruvate carboxykinase, and G-6-P in a concentration-dependent manner. The authors conclude that the tested extract inhibited glucose production through the activation of AMPK in HepG2 cells, and thus, the extract has potential in the treatment of T2DM. ${ }^{15}$ It is important to note that there are no previous reports in the literature supporting the traditional use of the plant in the treatment of T2DM or hypoglycemia.

\section{Cecropia obtusifolia Bertol (Cecropiaceae)}

Cecropia obtusifolia is a plant commonly used by the Mexican diabetic population to treat T2DM. Studies have assessed its hypoglycemic effect in animal models and in T2DM patients. The plant contains cholorogenic acid, an inhibitor of glucose6-phosphate translocase. The authors propose the mechanism by which the plant could produce the observed hypoglycemic effect with traditional use. They also test the hypothesis that targeting gluconeogenesis with an inhibitor of glucose-6phosphate translocase could result in a reduction of hepatic glucose production. For this purpose, they tested the effects of the plant on gluconeogenesis (in vivo) and on enzyme activity (in vitro). To test the inhibition of gluconeogenesis in vivo, a pyruvate tolerance test $(2 \mathrm{~g} / \mathrm{kg})$ was performed in 18-hour fasted n5-STZ rats. The effect of the extracts on G-6-P with a half-maximal inhibitory concentration of $224 \mathrm{~g} / \mathrm{mL}$ for C. obtusifolia aqueous, $160 \mathrm{~g} / \mathrm{mL}$ for C. obtusifolia butanolic, and $254 \mathrm{~g} / \mathrm{mL}$ for chlorogenic acid. The authors reached the conclusion that the results implied that the administration of the plant could improve glycemic control by blocking hepatic glucose output, especially in the fasting state. ${ }^{16}$

\section{Malmea depressa (Baill.) RE Fries (Annonaceae)}

This plant is traditionally used in the Mayan communities of Southeastern Mexico to treat T2DM. A root bark infusion is typically taken throughout the day between meals. The authors tested the inhibition of gluconeogenesis in vivo by performing a pyruvate tolerance test in n5-STZ rats after an 18-hour fasting period. The effects of the extracts on G-6-P activity were assayed in vitro with intact rat liver microsomes. In this work the ethanolic extract of the root bark dose-dependently inhibited a glucose peak in the animal model. The authors suggest that administration of the plant can improve glycemic control by blocking hepatic glucose production. ${ }^{17}$

\section{Melissa officinalis L (Lamiaceae)}

Lemon balm is a medicinal plant used in perfumes, cosmetics, tea, and food products in many countries. The medicinal uses have been cited as being a mild sedative, a spasmolytic and an antibacterial agent. The main components of the leaves are essential oils, including neral and geranial. The hypoglycemic effect of the plant was examined using male C57BL/ $\mathrm{KsJ}-\mathrm{db} / \mathrm{db}(\mathrm{db} / \mathrm{db})$ mice; the animals were fed normal chow or chow with $\mathrm{M}$. officinalis essential oil $(0.0125 \mathrm{mg} / \mathrm{day})$ for 6 weeks. Afterwards, an oral glucose tolerance test was performed. After 3 weeks of treatment, the glucose levels of the Melissa-treated group decreased substantially compared with the control group. Administration of the plant oils also improved glucose tolerance in $\mathrm{db} / \mathrm{db}$ mice. Blood glucose levels at 0,15 , and 120 minutes after glucose loading were significantly lower $(P<0.05)$ in the treated group. Key hepatic gluconeogenic genes were assayed by reverse transcription, (polymerase chain reaction amplification) and Western blotting. Melissa supplementation resulted in a significant decrease in G-6-P and in phosphoenolpyruvate carboxykinase mRNA levels when compared with the controls. It was concluded that the essential oils from Melissa decreased glucose concentrations by stimulating glucokinase activity and inhibiting G-6-P activity in the livers of $\mathrm{db} / \mathrm{db}$ mice. Key genes for carbohydrate and lipid homeostasis in the liver are regulated by insulin and glucose. Glucokinase catalyses the conversion of glucose into glucose-6-phosphate in the liver, thus playing a key role in the control of glucose homeostasis by supplying glucose-6-phosphate for glycogen storage, glycolysis or the pentose phosphate pathway. For these reasons, the glucokinase gene has been considered as a potential target for the pharmacological treatment of T2DM. ${ }^{18}$

\section{Salacia reticulata (Hippocrateaceae)}

This plant is used in Indian and Sri Lankan Ayurvedic traditional medicine as a specific remedy in the initial stages of diabetes. The aqueous extract from the root stems has been demonstrated to reduce fasting blood glucose levels in rats and mild T2DM patients. In the study the authors clarified the mechanisms by which an aqueous extract derived from the plant stems can reduce fasting glucose levels and, investigated hepatic gluconeogenesis in vivo and in vitro. The mechanism by which the plant extract reduced the fasting blood glucose levels in KK-Ay mice was explored. Gene expression profiles were assessed using DNA microarrays in samples from mice treated with the plant. It was confirmed that some genes were upregulated while others were downregulated. Among the genes associated with gluconeogenesis, the mRNA levels of FBP and G-6-P were downregulated compared with the control group. DNA microarray 
analysis revealed that the plant extract significantly decreased the mRNA levels of FBP and G-6-P, which are rate-limiting gluconeogenic enzymes in the liver of KK-Ay mice. Based on their experiments, it was concluded that the aqueous extract of Salacia reduces fasting blood glucose levels by downregulating the gluconeogenic pathway. ${ }^{19}$

\section{Satureja khuzestanica Jamzad (Lamiaceae)}

This plant is used in Southern Iran to treat diabetes and other illness. The evaluation of the hypoglycemic effect of the plant was performed in normal and in streptozotocin diabetic rats. The authors administered the essential oils at doses of 50 and $100 \mathrm{mg} / \mathrm{kg} / \mathrm{day}$ for 21 days. At the end of the experiment, the liver was removed, and the activities and gene expression of hepatic glycogen phosphorylase and phosphoenolpyruvate carboxykinase were evaluated. The plasma glucose concentrations of the diabetic treated rats compared with diabetic controls were significantly decreased. Hepatic glycogen phosphorylase activity and its mRNA levels in the diabetic rats treated with the plant extract were moderately increased. The activity of hepatic phosphoenolpyruvate carboxykinase and its mRNA levels were substantially decreased in normal rats. From these results, the authors state that the excessive inhibition of phosphoenolpyruvate carboxykinase in the liver of diabetic rats treated with Satureja may contribute to the plasma glucose lowering action through decreased gluconeogenesis, which appears to be related to the antioxidant properties of the plant extract. ${ }^{20}$

\section{Tinospora cordifolia (Willd.) Miers ex Hook $f$ and Thoms (Menispermaceae)}

This plant is used in India as a hypoglycemic. Its use to treat diabetes has been documented in the various systems of medicines that include Ayurveda, Siddha, and tribal medicines. It is used by many patients because of its commercial availability. The aqueous and alcoholic extracts of the root and stem show potent anti-hyperglycemic effects in animal models. In the present study, diabetes was induced by a combination of a high-fat diet for a period of 10 weeks followed by intraperitoneal injection of streptozotocin $(35 \mathrm{mg} / \mathrm{kg})$. Oral treatment of the extracts (100 and $200 \mathrm{mg} / \mathrm{kg}$ ) for 14 days regulated blood glucose in the liver, inhibited G-6-P and fructose 1,6-diphosphatase $(P<0.001)$ and restored glycogen content $(P<0.005)$, which was analyzed by histopathological staining with periodic acid-Schiff stain. The enzymes, G-6-P and fructose 1,6-diphosphatase, were analyzed following standard protocols. ${ }^{21}$

Another study evaluated the effects of the isoquinoline alkaloid-rich fraction derived from the stem of Tinospora cordifolia and three alkaloids: palmatine, jatrorrhizine, and magnoflorine (at doses of 5-80 $\mathrm{g} / \mathrm{mL}$ ) on hepatic gluconeogenesis in rat hepatocytes. ${ }^{22}$ The authors observed a significant inhibition $(P<0.001)$ of glucose production in hepatocytes when treated with the extract at concentrations of $20 \mathrm{~g} / \mathrm{mL}$ and above. The inhibition of gluconeogenesis was observed in a dose-dependent manner.

\section{Conclusion}

In recent years, the idea of inhibiting gluconeogenesis as a target to treat T2DM has attracted much interest. Because metformin is plant-derived, it would be logical to assume that medicinal plants could provide new therapeutic compounds. The search for these types of substances is only at the beginning. Basic research has been performed in the field, with researchers focusing on different mechanisms, including: the downregulation of mRNA levels of FBP and G-6-P; the activation of AMPK, which leads to a decrease of CREB, a key transcription factor for gluconeogenic enzyme phosphorylation; the expression of the glucokinase gene, which stimulates glucokinase activity and inhibits G-6-P; and the inhibition of phosphoenolpyruvate carboxykinase, which decreases gluconeogenesis and directly inhibits enzymatic activity of G-6-P and fructose 1,6-diphosphatase.

Current research is mainly performed in vitro through the exploration of enzymes or cell lines (hepatocytes) from rats or humans; only two studies reflect an in vivo situation in rats. It would be of interest for future studies to consider the whole picture and to generate a model of how a botanical could affect gluconeogenesis. "Pyruvate-loaded diabetic rats" appear to be a good in vivo model, whereas in vitro assays could be performed in a cell line to examine genetic expression and enzymatic activity.

We are certainly using a large variety of medicinal plants to treat diabetes and can estimate a number of reported plants used for this purpose. However, if we were to extrapolate the findings on a worldwide scale and consider that many plants have not yet been documented, it is difficult to calculate the exact number of plants with therapeutic potential. There must be many unknown plant species with potential medicinal properties. Since diabetes is a chronic disease, it requires ongoing treatment. For this reason, the continual consumption of a botanical that can inhibit the gluconeogenic pathway may be a good treatment strategy for people with T2DM.

\section{Acknowledgments}

This work was partially supported by "Dirección General de Asuntos del Personal Académico,UNAM", project 
IN228510, and “Consejo Nacional de Ciencia y Tecnología”, project CB 151264 .

\section{Disclosure}

The author declares no conflicts of interest in the writing and publication of this paper.

\section{References}

1. World Health Organization [homepage on the Internet]. Geneva: World Health Organization; [updated 2012; cited Dec 2011]. Available from: http://www.who.org. Accessed Dec 2011.

2. American Diabetes Association. Diagnosis and classification of diabetes mellitus. Diabetes Care. 2011;34(Suppl 1):S62-S69.

3. DeFronzo R, Mandarino L. Pathogenesis of type 2 diabetes mellitus. [article on the Internet]. 2009 [cited Dec 2011]. http://www.endotext. org. 2009. Accessed Dec 2011.

4. Hundal R, Krssak M, Dufour S, et al. Mechanism by which metformin reduces glucose production in type 2 diabetes. Diabetes. 2000;49(12): 2063-2069.

5. Guyton A, Hall J. Textbook of Medical Physiology. 11th ed. Philadelphia: Elsevier; 2006:836-839.

6. Tahrani AA, Bailey CJ, Del Prato S, Barnett AH. Management of type 2 diabetes: new and future developments in treatment. Lancet. 2011;378(9786):182-197.

7. Agius L. New hepatic targets for glycaemic control in diabetes. Best Pract Res Clin Endocrinol Metab. 2007;21(4):587-605.

8. He L, Sabet A, Djedjos S, Miller et al. Metformin and insulin suppress hepatic gluconeogenesis through phosphorylation of CREB binding protein. Cell. 2009;137(4):635-645.

9. Kim YD, Park KG, Lee YS, et al. Metformin inhibits hepatic gluconeogenesis through AMP-activated protein kinase-dependent regulation of the orphan nuclear receptor SHP. Diabetes. 2008;57(2):306-314.

10. Lee JM, Seo WY, Song KH, et al. AMPK-dependent repression of hepatic gluconeogenesis via disruption of CREB.CRTC2 complex by orphan nuclear receptor small heterodimer partner. J Biol Chem. 2010; 285(42):32182-32191.
11. Kirpichnikov D, McFarlane S, Sowers J. Metformin: an update. Ann Intern Med. 2002;137(1):25-33.

12. Zhou G, Myers R, Li Y, et al. Role of AMP-activated protein kinase in mechanism of metformin action. J Clin Invest. 2001;108(8): $1167-1174$.

13. Witters L. The blooming of the French lilac. J Clin Invest. 2001;108(8): 1105-1107.

14. Prabhakar P, Doble M. A target based therapeutic approach towards diabetes mellitus using medicinal plants. Curr Diabetes Rev. 2008;4(4): 291-308.

15. Yuan HD, Piao GC. An active part of Artemisia sacrorum Ledeb. suppresses gluconeogenesis through AMPK mediated GSK3 $\beta$ and CREB phosphorylation in human HepG2 cells. Biosci Biotechnol Biochem. 2011;75(6):1079-1984.

16. Andrade-Cetto A, Vázquez RC. Gluconeogenesis inhibition and phytochemical composition of two Cecropia species. J Ethnopharmacol. 2010;130(1):92-97.

17. Andrade-Cetto A. Inhibition of Gluconeogenesis by Malmeadepressa root. J Ethnopharmacol. 2011;137(1):930-933.

18. Chung MJ, Cho SY, Bhuiyan MJ, Kim KH, Lee SJ. Anti-diabetic effects of lemon balm (Melissa officinalis) essential oil on glucose- and lipidregulating enzymes in type 2 diabetic mice. Br J Nutr. 2010;104(2): 180-188.

19. Ima R, Mano H, Matsuura T, Nakatani S, Shimizu J, Wada M. Mechanisms of blood glucose-lowering effect of aqueous extract from stems of Kothala himbutu (Salacia reticulata) in the mouse. J Ethnopharmacol. 2009;121(12):234-240.

20. Shahsavari R, Ehsani-Zonouz A, Houshmand M, Salehnia A, Ahangari G, Firoozra M. Plasma glucose lowering effect of the wild Satureja khuzestanica Jamzad essential oil in diabetic rats: role of decreased gluconeogenesis. Pak J Biol Sci. 2009;12(2):140-145.

21. Sangeetha MK, Blaji Raghavendran HR, Gayathri V, Vasanthi HR. Tinospora cordifolia attenuates oxidative stress and distorted carbohydrate metabolism in experimentally induced type 2 diabetes in rats. J Nat Med. 2011;65(3-4):544-550.

22. Patel M, Mishra S. Hypoglycemic activity of alkaloidal fraction of Tinosporacordifolia. Phytomedicine. 2011;18(12):1045-1052.
Botanics: Targets and Therapy

\section{Publish your work in this journal}

Botanics: Targets and Therapy is an international, peer-reviewed, open access journal focusing on the discovery and development of active compounds based upon or found naturally occurring in the plant kingdom that may have therapeutic potential in any disease state. The manuscript management system is completely online and includes a very

Submit your manuscript here: http://www.dovepress.com/botanics-targets-and-therapy-journal

\section{Dovepress}

quick and fair peer-review system. Visit http://www.dovepress.com/ testimonials.php to read real quotes from published authors. 